\title{
Chaperone co-inducer BGP-15 inhibits histone deacetylases and enhances the heat shock response through increased chromatin accessibility
}

\author{
Marek A. Budzyński ${ }^{1,2} \cdot{\text { Tim } \text { Crul }^{3} \text { (D) } \cdot \text { Samu V. Himanen }}^{1,2} \cdot$ Noemi Toth $^{3} \cdot$ \\ Ferenc Otvos ${ }^{3} \cdot$ Lea Sistonen ${ }^{1,2} \cdot{\text { Laszlo } \text { Vigh }^{3}}^{3}$
}

Received: 3 February 2017 / Revised: 22 March 2017 / Accepted: 7 April 2017 / Published online: 4 May 2017

(C) The Author(s) 2017. This article is an open access publication

\begin{abstract}
Defects in cellular protein homeostasis are associated with many severe and prevalent pathological conditions such as neurodegenerative diseases, muscle dystrophies, and metabolic disorders. One way to counteract these defects is to improve the protein homeostasis capacity through induction of the heat shock response. Despite numerous attempts to develop strategies for chemical activation of the heat shock response by heat shock transcription factor 1 (HSF1), the underlying mechanisms of drug candidates' mode of action are poorly understood. To lower the threshold for the heat shock response activation, we used the chaperone co-inducer BGP15 that was previously shown to have beneficial effects on several proteinopathic disease models. We found that BGP15 treatment combined with heat stress caused a substantial increase in HSF1-dependent heat shock protein 70 (HSPA1A/ B) expression already at a febrile range of temperatures. Moreover, BGP-15 alone inhibited the activity of histone deacetylases (HDACs), thereby increasing chromatin
\end{abstract}

Marek A. Budzyński and Tim Crul contributed equally to this study.

Electronic supplementary material The online version of this article (doi:10.1007/s12192-017-0798-5) contains supplementary material, which is available to authorized users.

Lea Sistonen

lea.sistonen@abo.fi

Laszlo Vigh

vigh@brc.hu

1 Faculty of Science and Engineering, Cell Biology, Åbo Akademi University, FI-20520 Turku, Finland

2 Turku Centre for Biotechnology, Åbo Akademi University and University of Turku, FI-20520 Turku, Finland

3 Biological Research Centre of the Hungarian Academy of Sciences, Szeged 6726, Hungary accessibility at multiple genomic loci including the stressinducible HSPA1A. Intriguingly, treatment with well-known potent HDAC inhibitors trichostatin A and valproic acid enhanced the heat shock response and improved cytoprotection. These results present a new pharmacological strategy for restoring protein homeostasis by inhibiting HDACs, increasing chromatin accessibility, and lowering the threshold for heat shock response activation.

Keywords Heat shock factor protein $1(\mathrm{HSF} 1) \cdot$ Histone deacetylase (HDAC) - Stress response · Transcription · TSA . VPA

\section{Introduction}

Proper protein function is critical for all organisms. When exposed to stress, such as changes in ambient temperature, UV radiation, or in many pathologies, organisms trigger survival mechanisms to maintain their protein homeostasis. One such survival mechanism is the heat shock response (HSR) that consists of a complex network of inducible molecular chaperones, including heat shock proteins (Hsps), which are under the transcriptional control of heat shock factor 1 (HSF1) (Budzyński and Sistonen 2017). Under normal conditions, Hsps assist nascent proteins to reach their final conformation, whereas, upon exposure to stress, Hsps bind to misfolded proteins to prevent the formation of aggregates and either facilitate their refolding or direct them to degradation (Hartl et al. 2011). Failure to adequately mount the HSR is central to many severe and prevalent pathological conditions, such as neurodegenerative diseases, muscle dystrophies, and metabolic disorders (Chung et al. 2008; Gehrig et al. 2012; Su and Dai 2016). Considering the immense impact of these diseases in the society, therapeutic strategies to restore the HSR are 
urgently needed. As genetic approaches are not feasible on a human scale, several pharmacological approaches to directly target the pathway of HSF1-mediated HSR have been tested in various disease models (Neef et al. 2010; Calamini et al. 2011; West et al. 2012). For instance, activation of HSF1 by the HSPC1 (Hsp90) inhibitor 17-(allylamino)geldanamycin (17-AAG) ameliorates cytotoxicity in an Alzheimer's disease model (Chen et al. 2014), and activation of HSF1 by celastrol was shown to reduce toxicity in a cardiomyopathy model (Sharma et al. 2014). However, most of the currently used pharmacological activators of the HSR have cytotoxic effects which severely hamper the drug development.

The hydroximic acid derivative BGP-15 is a small ( $350 \mathrm{Da})$ multi-target molecule, which intercalates into membranes and stabilizes their lipid rafts, reduces the levels of reactive oxygen species by enhancing mitochondrial efficiency, and inhibits both poly(adenosine 5 '-diphosphate)-ribose]polymerase 1 (PARP-1) and tumour necrosis factor- $\alpha$-induced pathways (Chung et al. 2008; Gombos et al. 2011; Henstridge et al. 2014; Gungor et al. 2014; Sumegi et al. 2017; Crul et al. 2013). Multiple studies have shown beneficial effects of BGP-15 on proteinopathic disease models, such as insulin resistance (Henstridge et al. 2014; Literáti-Nagy et al. 2014), atrial fibrillation (Zhang et al. 2011), muscle dystrophy (Gehrig et al. 2012; Kennedy et al. 2016), and ventilationinduced diaphragm dysfunction (VIDD) (Salah et al. 2016). In these cases, the BGP-15-mediated improvement of protein homeostasis and survival was reported to be due to its capacity to co-induce Hsps. For instance, BGP-15 treatment resulted in HSPA1A/B (Hsp70) upregulation in rat and rabbit models of insulin resistance, dystrophic mice, and the VIDD rat model (Henstridge et al. 2014; Literáti-Nagy et al. 2014; Salah et al. 2016), while upregulation of DmHSP23 was observed in the Drosophila tachycardia model (Zhang et al. 2011). Based on these findings, the term "membrane lipid therapy" pharmaceuticals was introduced as a molecular base for drug discovery and disease treatment through the modulation of cell membrane composition and structure using BGP-15 and other hydroximic acid derivatives (Escribá et al. 2015).

During the activation-attenuation cycle, HSF1 is extensively post-translationally modified, binds to DNA, activates gene transcription, and is subsequently released from its target sites (Hietakangas et al. 2003; Westerheide et al. 2009; Budzyński and Sistonen 2017; Raychaudhuri et al. 2014; Budzyński et al. 2015). Previously, it was reported that the hydroxylamine derivative bimoclomol enhances the expression of Hsps and has a cytoprotective effect upon several stresses including ischaemia (Vígh et al. 1997). Mechanistically, bimoclomol has been shown to bind to HSF1, thereby prolonging HSF1 DNAbinding activity (Hargitai et al. 2003). In this study, we investigated whether the chaperone co-inducing capacity of BGP15 , similarly to bimoclomol, stems from changes in the activation-attenuation cycle of HSF1. We found, however, that BGP-15 accelerates the activation and attenuation of HSF1 upon stress, sensitizes HSF1 by lowering its activation threshold and facilitating Hsp expression at a febrile range of temperatures. Surprisingly, BGP-15 alone inhibits the activity of histone deacetylases (HDACs), resulting in increased chromatin accessibility at multiple genomic loci, including HSPA 1A. Using well-known potent HDAC inhibitors (trichostatin A and valproic acid), we demonstrate that HDAC inhibition enhances the HSR and provides cytoprotection against proteotoxic insults. Taken together, we propose a new strategy for chemical activation of HSF1, by increasing chromatin accessibility through HDAC inhibition, which subsequently sensitizes and accelerates HSF1 activation under physiological stress conditions.

\section{Methods}

Cell culture and treatments Mouse embryonic fibroblasts (MEFs) were cultured in high-glucose Dulbecco's modified Eagle's medium containing 10\% foetal calf serum, $2 \mathrm{mM} \mathrm{L}-$ glutamine, streptomycin $(100 \mathrm{~g} / \mathrm{ml})$, penicillin $(100 \mathrm{U} / \mathrm{ml})$, and $1 \times$ MEM non-essential amino acid solution. Heat shock treatments were conducted in a water bath at 40,42 , and $45^{\circ} \mathrm{C}$ (for details, see figure legends). BGP-15 was purchased from N-Gene. Trichostatin A (TSA) was dissolved in DMSO, and valproic acid (VPA) and nicotinamide (NAM) were dissolved in water.

Quantitative RT-PCR (qRT-PCR) Immediately after treatment, RNA was isolated with RNeasy Mini Kit (Qiagen) according to the manufacturer's instructions and quantified using a NanoDrop ND-1000 spectrophotometer (Thermo Scientific). One microgram of total RNA was reverse transcribed with the iScript kit (Bio-Rad). KAPA PROBE FAST ABI Prism qPCR Kit (Kapa Biosystems) and SensiFAST SYBR Hi-ROX (Bioline Reagents) were used for qRT-PCRs that were performed with StepOnePlus or QuantStudio 12K Flex Real-Time PCR System. List of the primers and probes used for qRT-PCR is in Supplementary Table S1. Relative quantities of the target gene messenger RNAs (mRNAs) were normalized against their respective 18S RNA (RNA18S5). All reactions were run in triplicate from samples derived from at least four biological replicates.

Chromatin immunoprecipitation (ChIP) A total of $3 \times 10^{7}$ MEFs were cross-linked immediately after treatment on ice for $10 \mathrm{~min}$ with a final concentration of $1 \%$ formaldehyde, followed by 5 min quenching in $125 \mathrm{mM}$ glycine. After lysis in Joost's lysis buffer (1\% SDS, 10 mM EDTA, 50 mM Tris$\mathrm{HCl}[\mathrm{pH} 8.1])$ supplemented with $1 \times$ Pierce Protease Inhibitor (Thermo Scientific), $0.5 \mathrm{mM}$ PMSF, and $0.2 \mathrm{mM}$ sodium orthovanadate, samples were sonicated for $10 \mathrm{~min}$ using a 
Diagenode Bioruptor, and $1 \mathrm{mg}$ of whole-cell extracts was used for each immunoprecipitation. Samples were precleared with $50 \%$ slurry protein G-Sepharose beads (GE Healthcare Life Sciences) saturated with BSA and salmon sperm DNA, and immunoprecipitation was performed overnight at $4{ }^{\circ} \mathrm{C}$ using antibodies against HSF1 (SPA-901; Enzo Life Sciences). Normal rabbit serum was used as a nonspecific antibody control. After washing of the immunocomplexes, the remaining RNA and proteins were digested by using RNase $\mathrm{A}$ and proteinase $\mathrm{K}$, respectively. Cross-links were reversed by incubating the samples overnight at $65{ }^{\circ} \mathrm{C}$. DNA was purified with phenol-chloroform. Samples were analysed by qPCR using QuantStudio $12 \mathrm{~K}$ Flex Real-Time PCR System (Applied Biosystems). List of the primers used for qPCR is in Supplementary Table S2. Values obtained from non-specific antibody control were subtracted from the immunoprecipitation samples, which were then normalized to values obtained for input samples.

Nuclear fractionation and HDAC activity assay $8 \times 10^{6}$ MEFs were used for HDAC activity assay. Cells were collected in cold PBS, and nuclear fractionation was conducted using the NE-PER Nuclear and Cytoplasmic Extraction Reagents kit (Thermo Fisher Scientific) according to the manufacturer's protocol. HDAC activity was measured using a commercially available, non-radioactive HDAC activity assay kit (Active Motif), according to the manufacturer's instructions. Briefly, $40 \mu \mathrm{g}$ of nuclear lysates were incubated in HDAC assay buffer containing BOC-(Ac)Lys-pNi-troanilide for $60 \mathrm{~min}$ at $37^{\circ} \mathrm{C}$. The reaction was stopped by adding the stop solution, and after adding the complete developing solution, the mixture was incubated for another $15 \mathrm{~min}$ at room temperature. Absorbance was measured at $405 \mathrm{~nm}$ using the Hidex Sense microplate reader.

In vitro HDAC activity assay The inhibitory effect of BGP-15 on HDAC 1, 4, 6, and 10 and SIRT1 was determined using full-length recombinant HDACs and a fluorogenic substrate (both from BPS Bioscience) according to the manufacturer's instructions. Briefly, the fluorogenic substrate was incubated with purified HDACs with various concentrations of BGP-15, TSA (for HDAC 1, 4, 6, and 10), or NAM (for SIRT1) for $30 \mathrm{~min}$ at $37{ }^{\circ} \mathrm{C}$. The reaction was stopped by adding the developing solution, and the mixture was incubated for another $15 \mathrm{~min}$ at room temperature. Fluorescence intensity was measured with excitation and emission at a wavelength of 355/460 $\mathrm{nm}$ using the Hidex Sense microplate reader. Blank values were subtracted from the sample values, and the samples without an inhibitor were set to value 100 . All reactions were run in quadruple using two different aliquots of inhibitors.
Micrococcal nuclease (MNase) assay MNase assay was modified from a previously described protocol (Elsing et al. 2014). $1.5 \times 10^{7}$ MEFs were cross-linked with $1 \%$ formaldehyde by incubating cells for $10 \mathrm{~min}$ at $37^{\circ} \mathrm{C}$, after which $125 \mathrm{mM}$ glycine was added for $5 \mathrm{~min}$ at $4{ }^{\circ} \mathrm{C}$. Cell pellets were washed and resuspended in TM2 buffer $(10 \mathrm{mM}$ Tris [pH 7.5], $2 \mathrm{mM} \mathrm{MgCl} 2,1 \mathrm{mM}$ DTT, $5 \mathrm{mM}$ PMSF, and $1 \times$ Pierce Protease Inhibitor). Samples were divided into two aliquots: one was digested with MNase (New England Biolabs), and the other was sonicated for 12 min using a Bioruptor (Diagenode). Samples were incubated with MNase at a final concentration of $6.3 \mathrm{U} / \mu \mathrm{l}$ for $10 \mathrm{~min}$ at $37^{\circ} \mathrm{C}$, after which the reaction was stopped by adding $5 \%$ SDS and $50 \mathrm{mM}$ EGTA. $0.2 \mathrm{M} \mathrm{NaCl}$ was added, and cross-links were reversed by incubating samples at $65{ }^{\circ} \mathrm{C}$ overnight. Samples were treated with RNase A $(6 \mu \mathrm{g} / \mathrm{ml})$ and proteinase K $(50 \mu \mathrm{g} / \mathrm{ml})$. DNA was purified with phenol-chloroform. Samples were analysed by qPCR QuantStudio 12K Flex Real-Time PCR System. List of the primers used for qPCR is in Supplementary Table S2. The enrichment of MNase-digested DNA was normalized to sonicated DNA. Values were compared with non-treated MNase resistance for the transcriptional start site (TSS) region of each of the analysed loci which was set to value 1 .

Cell viability assay MEFs were grown on 96-well white, clear-bottom, tissue culture plates (PerkinElmer) at density $5 \times 10^{3}$ cells per well. Cells were either exposed to heat shock at 42 or $45{ }^{\circ} \mathrm{C}$ (for details, see figure legends) and left to recover for $16 \mathrm{~h}$ at $37^{\circ} \mathrm{C}$ or left untreated. Culture media were aspirated, $1 \times$ Calcein AM (Invitrogen) diluted in PBS was added to the cells, and samples were incubated for $30 \mathrm{~min}$ at $37{ }^{\circ} \mathrm{C}$. Fluorescence intensity was measured with excitation and emission at a wavelength of 485/535 nm using th Hidex Sense microplate reader. Blank values were subtracted from the sample values, and the cell death of non-treated non-heatshocked samples was set to value 0 . All reactions were run in quadruple.

Statistical analysis All statistical analyses were performed using GraphPad Prism 7 software with tests as indicated in the figure legends.

\section{Results}

BGP-15 accelerates the activation of HSF1 To investigate whether BGP-15, similarly to bimoclomol (Hargitai et al. 2003), enhances the HSR through delaying HSF1 attenuation, we treated MEFs with $10 \mu \mathrm{M}$ BGP- 15 for $1 \mathrm{~h}$ at $37^{\circ} \mathrm{C}$ followed by exposure to $42{ }^{\circ} \mathrm{C}$ for $60,90,120$, and $180 \mathrm{~min}$ and analysed the HSPA $1 A / B(H s p 70)$ and DNAJB1 (Hsp40) mRNA levels. During the course of heat exposure, BGP-15 co-induced the expression of $H S P A 1 A / B$ and DNAJB1 mRNA 
up to $90 \mathrm{~min}$, resulting in at least $20 \%$ increase in the mRNA levels (Fig. 1a). Surprisingly, at 120 min, BGP-15-treated cells exhibited a reduction in the mRNA levels of $H S P A 1 A / B$ and $D N A J B 1$, when compared to cells exposed to heat shock alone, indicating that HSF1 might attenuate faster in the presence of BGP-15. However, in cells exposed to heat stress for 180 min, the mRNA levels of Hsp70 and Hsp40 were similar in both BGP-15- and non-treated cells. These observations suggest that, unlike bimoclomol, BGP-15 does not delay the HSF1 attenuation; rather, it enhances Hsp expression in the early phase of HSR.

To verify whether BGP-15 treatment affects HSF1 DNAbinding activity in the context of chromatin, we used chromatin immunoprecipitation assay (ChIP) and compared the occupancy of HSF1 in the presence and absence of BGP-15 at the HSPA1A (Hsp 70) promoter region containing both the proximal and distal heat shock elements (Perry et al. 1994). MEFs were treated as above followed by immunoprecipitation with an HSF1 antibody or normal rabbit serum as a nonspecific antibody. Under control conditions, the signal for the occupancy of HSF1 at the HSPA1A promoter was hardly detectable in both non-treated and BGP-15-treated cells (Fig. 1b), showing that BGP-15 alone does not induce the DNA-binding activity of HSF1. Upon heat stress, the occupancy of HSF1 increased, and in the BGP-15-treated samples, we observed a $200 \%$ higher binding of HSF 1 at the $60 \mathrm{~min}$ of heat shock when compared to heat shock alone (Fig. 1b), which corresponds to the higher expression levels of Hsps in BGP-15-treated cells (Fig. 1a). However, already at $90 \mathrm{~min}$, HSF1 binding had reduced by $50 \%$ in the BGP-15-treated cells when compared to the cells exposed to heat shock alone (Fig. 1b). The HSF1 binding to the HSPA1A promoter further decreased at 120 and $180 \mathrm{~min}$ of heat stress in both BGP-15and non-treated cells, indicating HSF1 attenuation from the promoter. These results demonstrate that the potentiating effect of BGP-15 on the HSF1 DNA-binding activity is transient and that BGP-15 accelerates the activation phase of the HSF1 cycle.

To study the HSF1 activation kinetics in more detail, we exposed BGP-15-treated and non-treated MEFs to $42{ }^{\circ} \mathrm{C}$ heat shock for 15 and $30 \mathrm{~min}$ and analysed the $H S P A 1 A / B$ and DNAJB1 mRNA levels and HSF1 binding to the HSPA1A promoter. As expected, BGP-15 treatment resulted in increased expression of Hsps during the early phase of HSR (Fig. 1c). In addition, the enhanced HSF1 binding to the HSPA1A promoter was observed already at $15 \mathrm{~min}$ of heat shock (Fig. 1d). These data further confirm that BGP-15 accelerates activation of HSF1 upon proteotoxic stress.

Since in many proteinopathic diseases the HSR is not mounted (Gehrig et al. 2012), we examined whether BGP15 can activate HSF1-mediated HSR in the context of mild stress. For this purpose, we exposed MEFs to a febrile temperature of $40^{\circ} \mathrm{C}$ for 15,30 , and $60 \mathrm{~min}$. This temperature was sufficient to activate the HSR, although the kinetics were delayed and the magnitude of the $H S P A 1 A / B$ induction was lower than at $42{ }^{\circ} \mathrm{C}$ (Fig. 1e vs. Fig. 1c). Intriguingly, in the presence of BGP-15, Hsp70 mRNA levels increased by 2.2fold already at a 15-min heat shock, while in the non-treated cells, there was no change in the levels of $H S P A 1 A / B$ mRNA (Fig. 1e). These results indicate that BGP-15 sensitizes HSF1 by lowering the threshold for its activation.

BGP-15 inhibits HDAC activity The mammalian family of histone deacetylases (HDACs) consists of 18 enzymes, of which 11 are zinc-dependent (classes I, II, and IV) and seven require the $\mathrm{NAD}^{+}$co-factor for activity (class III) (Roche and Bertrand 2016). HDACs prevent acetylation of histones and many non-histone proteins including transcription factors. Previously, it has been shown that the activity of HDACs regulates duration and magnitude of HSF1 binding to DNA (Westerheide et al. 2009; Zelin and Freeman 2015). Hence, it is plausible that BGP-15 modulates HSF1 DNA-binding activity through interfering with HDACs. Therefore, we analysed the activity of class I and II HDACs in the presence of BGP-15. First, we treated MEFs with $10 \mu \mathrm{M}$ BGP-15 for $1 \mathrm{~h}$ or $10 \mu \mathrm{M}$ trichostatin A (TSA) for $4 \mathrm{~h}$. TSA is a wellknown class I and II HDAC inhibitor (Yoshidas et al. 1990), which has been shown to effectively inhibit HDACs in MEFs after 2 to $4 \mathrm{~h}$ of treatment (Manova et al. 2012). The activity of class I and II HDACs in the nuclear extracts was assessed using a commercially available assay (for details, see "Methods"). As expected, the pre-treatment of cells with TSA resulted in a $30 \%$ reduction in the activity of HDACs compared to control cells (Fig. 2a). Intriguingly, cells treated with BGP-15 showed also decreased activity of HDACs (15\% reduction).

The activity of HDAC enzymes is regulated through several mechanisms, i.e. signalling pathways and interacting proteins (Sengupta and Seto 2004). Previously, the only direct effect of BGP-15 has been observed on the lipid rafts in the plasma membrane (Gombos et al. 2011). As lipid rafts are nano-scaled domains in the plasma membrane which cluster signalling proteins (Simons and Toomre 2000), BGP-15 might modulate plasma membrane originating signalling cascades which could ultimately also affect HDAC activity. To exclude the possibility that the plasma membrane originating signalling cascades are involved in BGP-15-mediated HDAC inhibition, we performed an HDAC activity assay on isolated nuclei. For this purpose, nuclear extracts from MEFs were incubated with $10 \mu \mathrm{M}$ BGP-15 or $1 \mu \mathrm{M}$ TSA for $1 \mathrm{~h}$ at $37{ }^{\circ} \mathrm{C}$, and the HDAC activity was measured as above. As shown in Fig. 2b, both BGP-15 and TSA equally inhibited the HDAC activity (35\% reduction). These results suggest that BGP-15 inhibits the activity of nuclear HDACs independently of plasma membrane originating signalling cascades. 
Fig. 1 BGP-15 accelerates the activation of HSF1. a-d MEFs were treated with or without $10 \mu \mathrm{M}$ BGP-15 for $1 \mathrm{~h}$, and then exposed to heat shock at $42^{\circ} \mathrm{C}$ for indicated times, or left at $37^{\circ} \mathrm{C}$ (C). a, $\mathbf{c}$ The mRNA levels of $H S P A 1 A / B(H s p 70)$ and DNAJB1 (Hsp40) were quantified using qRT-PCR and normalized against RNA18S5. The values are shown relative to the respective mRNA levels in the non-treated cells in control conditions $(C)$, which was arbitrarily set to value 1 . The data are presented as mean values from four independent experiments plus the SEM. b, d The occupancy of HSF1 at the HSPA1A (Hsp 70) promoter was analysed by ChIP, followed by qPCR. qPCR values of the immunoprecipitations were normalized to the input values. The data are presented as mean values from four independent experiments plus the SEM. e MEFs were treated with or without $10 \mu \mathrm{M}$ BGP-15 for $1 \mathrm{~h}$, and then exposed to mild heat stress at $40^{\circ} \mathrm{C}$ for 15,30 , and $60 \mathrm{~min}$, or left at $37^{\circ} \mathrm{C}(\mathrm{C})$. The mRNA levels of $H S P A 1 A / B$ $(H s p 70)$ were quantified as in $\mathbf{a}$. Statistical analyses in a-e were performed using one-way ANOVA with Holm-Sidak post hoc test. $n s$ non-significant. $* P \leq 0.05 ; * * * P \leq 0.001$
A

HSPA1A/B
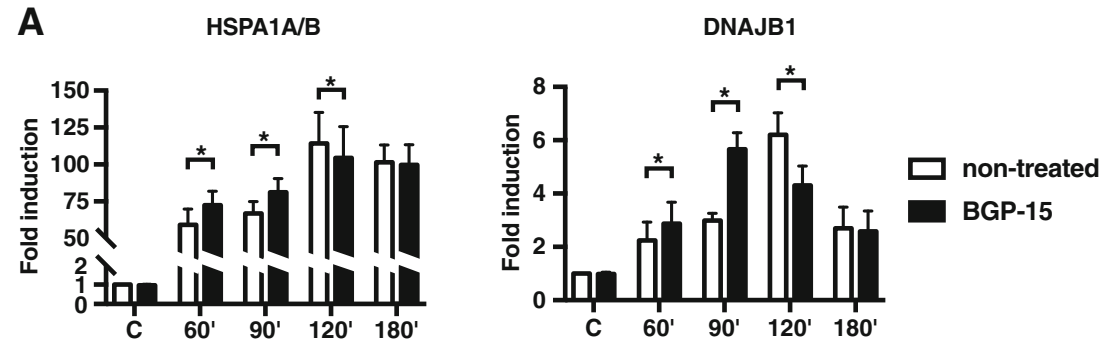

B
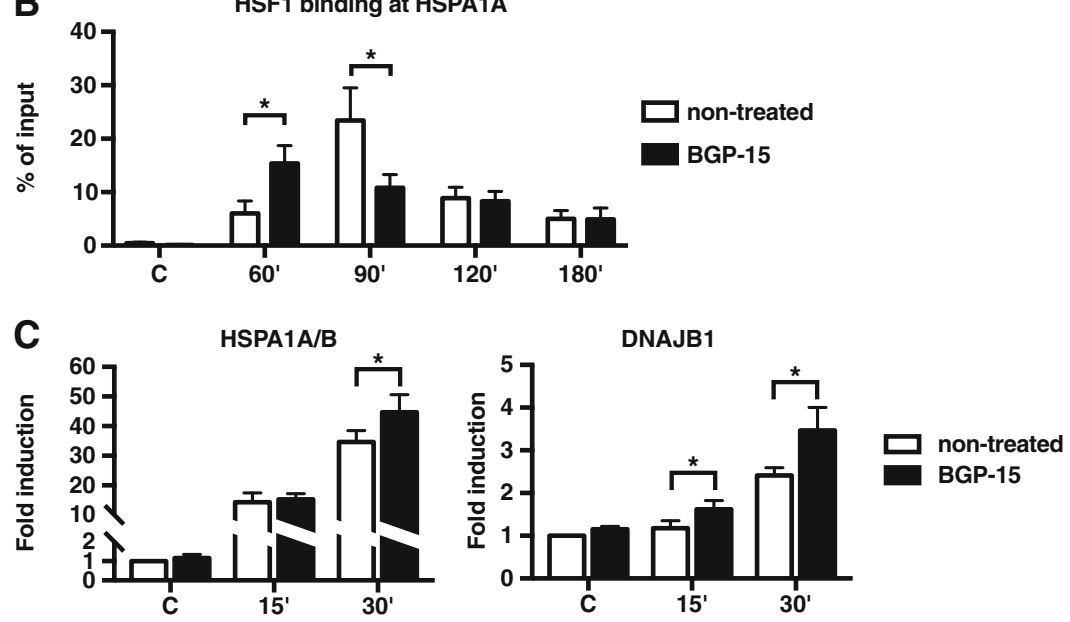

D
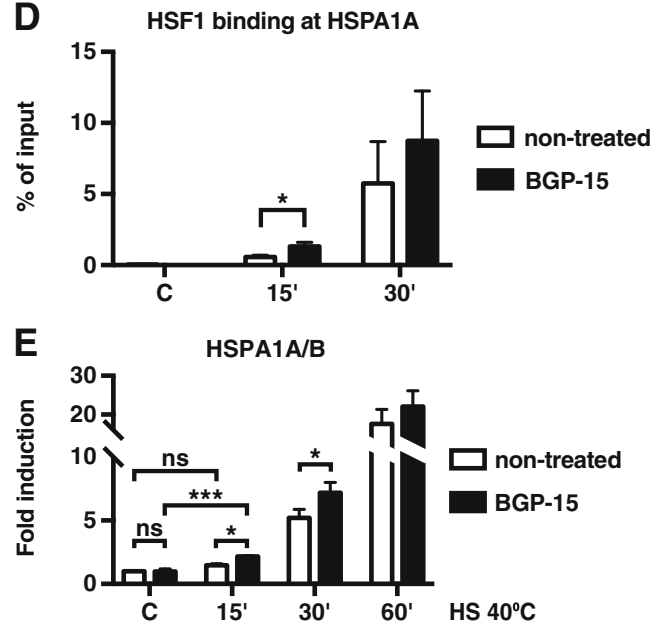

To gain insight, whether BGP-15 is a direct HDAC inhibitor, we performed an HDAC activity assay with an acetylated fluorogenic substrate and purified HDACs belonging to class I (HDAC1), II (HDAC4, 6, and 10), and III (SIRT1). We did not test HDAC11, a sole member of class IV, since its expression is limited to the muscle, brain, and kidney (Gao et al. 2002). We measured the deacetylation of a substrate in the presence of BGP-15 or the potent HDAC inhibitors TSA or nicotinamide (NAM) (Bitterman et al. 2002). As expected, TSA inhibited the activity of class I and II HDACs (Fig. 2c), while NAM inhibited the activity of SIRT1 (Fig. 2d). However, BGP-15 was unable to inhibit the tested HDACs (Fig. 2c, d), demonstrating that BGP-15 is capable of inhibiting HDACs in cells, but it is not a direct HDAC inhibitor.

BGP-15 increases chromatin accessibility Accessibility of chromatin, including nucleosome assembly and disassembly, is regulated by dynamic acetylation-deacetylation cycles of histones and other chromatin-associated proteins (Henikoff and Shilatifard 2011). HDAC inhibition results in protein hyperacetylation followed by chromatin remodelling and alterations in gene expression (Delcuve et al. 2012). Considering that BGP-15 inhibits the activity of HDACs (Fig. 2), we examined the effect of BGP-15 on the chromatin structure of the promoter and coding region of HSPA1A. 

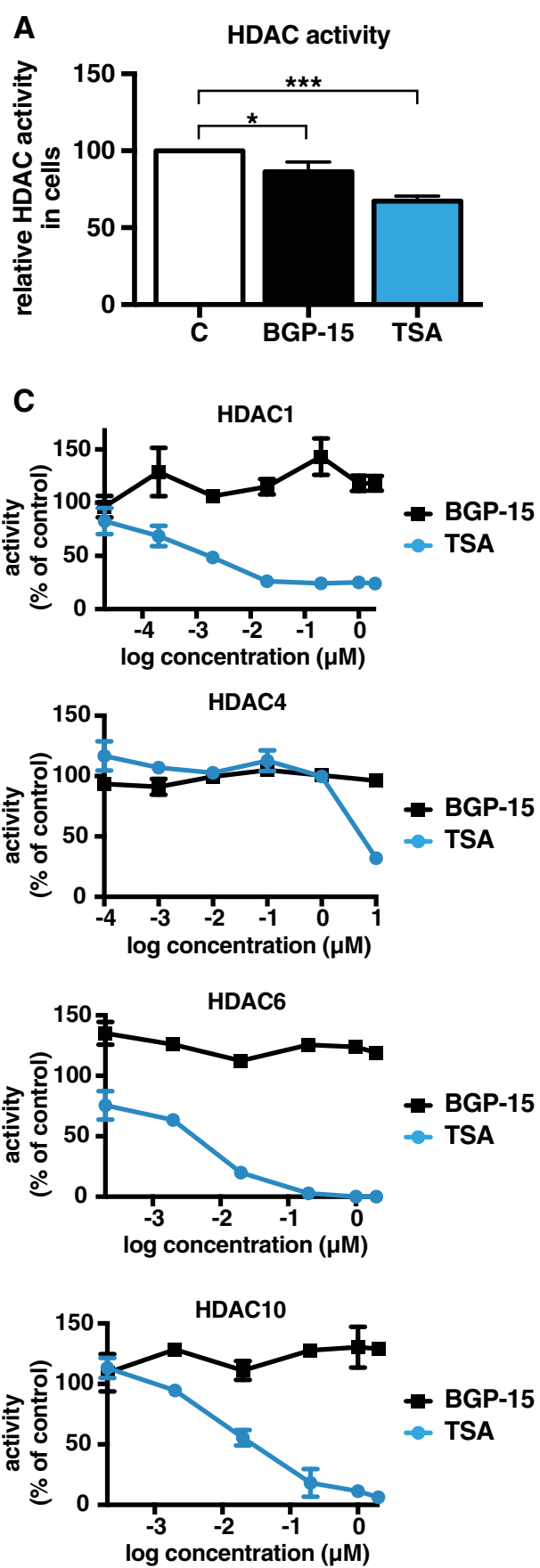

Fig. 2 BGP-15 inhibits HDAC activity. HDAC activity upon BGP-15 treatment was assessed both in cells (a) and in isolated nuclei (b). a MEFs were treated with either $10 \mu \mathrm{M}$ BGP-15 for $1 \mathrm{~h}, 10 \mu \mathrm{M}$ trichostatin A $(T S A)$ for $4 \mathrm{~h}$, or DMSO as a vehicle control $(C)$. Nuclear fractions were isolated and HDAC activity was assessed. HDAC activity in DMSOtreated samples was set to value 100 . The data are presented as mean values from at least four independent experiments plus the SEM. b Nuclear fractions from MEFs were isolated and treated with either $10 \mu \mathrm{M}$ BGP-15 or $1 \mu \mathrm{M}$ TSA or left untreated $(C)$, and HDAC activity was assessed. The data are presented as mean values from four independent experiments plus the SEM, where HDAC activity in untreated samples was set to value 100. Statistical analyses for $\mathbf{a}$ and $\mathbf{b}$ were performed using one-way ANOVA with Holm-Sidak post hoc test. $* P \leq 0.05$; $* * * P \leq 0.001$. c Purified HDAC $1,4,6$, and 10 were incubated with
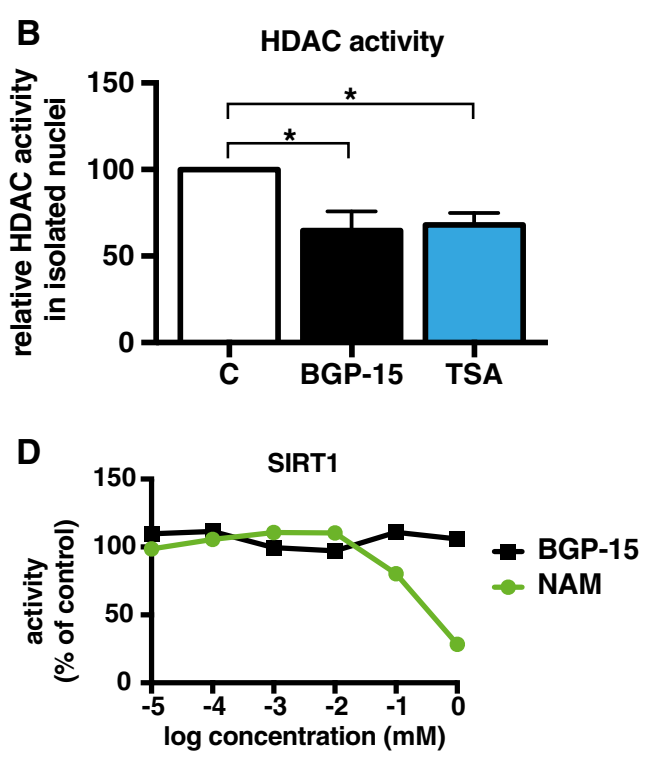

1:10 serial dilutions of BGP- 15 or TSA for $30 \mathrm{~min}$ at $37{ }^{\circ} \mathrm{C}$ in the presence of an acetylated fluorogenic substrate. All reactions were run in quadruple. The HDAC activity was quantified as a change in fluorescence intensity of the substrate. HDAC activity in the samples without inhibitors was set to value 100 . The data are presented as mean values from two independent experiments \pm the SEM. The IC50 values for TSA are $1 \mathrm{nM}$ for HDAC1, $1600 \mathrm{nM}$ for HDAC4, $8 \mathrm{nM}$ for HDAC6, and $14 \mathrm{nM}$ for HDAC10. d Purified SIRT1 was incubated with 1:10 serial dilution of BGP-15 or NAM for 30 min at $37{ }^{\circ} \mathrm{C}$ in the presence of an acetylated fluorogenic substrate. All reactions were run in quadruple. The SIRT1 activity was quantified as a change in fluoresce intensity of the substrate. SIRT1 activity in the samples without inhibitors was set to value 100 . The data are presented as mean values from two independent experiments \pm the SEM. The IC50 value for NAM is $108 \mu \mathrm{M}$ 


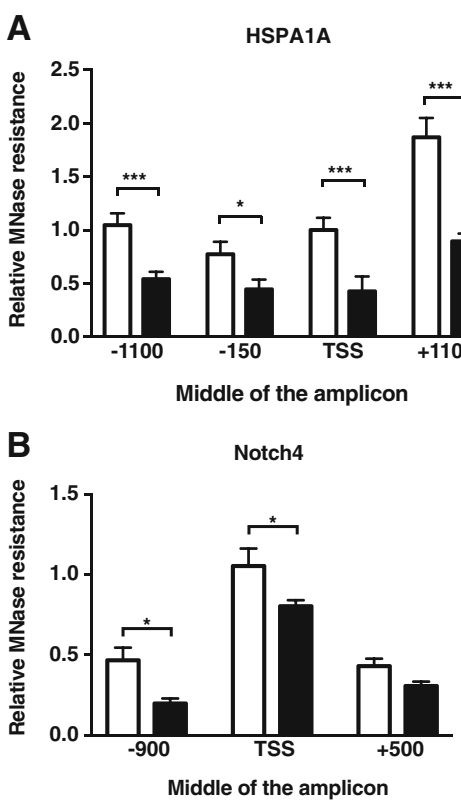

Fig. 3 Treatment with BGP-15 results in increased chromatin accessibility. Chromatin structure for genomic region of HSF1 target gene HSPA1A $(H s p 70)$ (a) and two HSR-unrelated genomic regions: Notch4 (Neurogenic locus notch homologue protein) (Chitnis and Balle-Cuif 2016) and Daxx (Death domain associated protein) (Lindsay et al. 2008) (b) was analysed using micrococcal nuclease (MNase) sensitivity assay. MEF cells were treated with or without $10 \mu \mathrm{M} \mathrm{BGP}-15$ for $1 \mathrm{~h}$, and chromatin accessibility was assessed using MNase assay followed by
Daxx

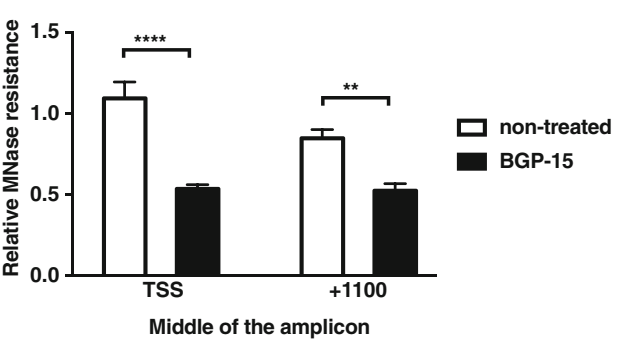

qPCR. The qPCR values obtained from the MNase-treated samples were related to values obtained from sonicated samples and normalized to the non-treated transcriptional start site (TSS) region, which was arbitrarily set to value 1 . The data are presented as mean values from four independent experiments plus the SEM. Statistical analysis was performed using multiple $t$ tests with Holm-Sidak post hoc test. $* P \leq 0.05 ; * * P \leq 0.01$; $* * * P \leq 0.001 ; * * * * P<0.0001$

impact of BGP-15 and two well-known potent HDAC inhibitors, TSA and valproic acid (VPA) (Göttlicher et al. 2002) on Hsp expression. We treated MEFs with either $10 \mu \mathrm{M}$ BGP-15, $10 \mu \mathrm{M}$ TSA, or $500 \mu \mathrm{M}$ VPA for $1 \mathrm{~h}$ at $37^{\circ} \mathrm{C}$ followed by exposure to $42{ }^{\circ} \mathrm{C}$ for 30 and $60 \mathrm{~min}$. As shown in Fig. 4a, the HDAC inhibition did not result in elevated mRNA levels of $H S P A 1 A / B$ or DNAJB1 under normal conditions. However, upon heat stress, BGP-15, TSA, and VPA exhibited chaperone coinducing capacity. Elevated expression of Hsps upon HDAC inhibition should yield cytoprotective effect upon stress. To test whether the HDAC inhibition has an impact on cell survival during proteotoxic conditions, we measured cell death in MEFs pre-treated with BGP-15, TSA, or VPA for $1 \mathrm{~h}$ and exposed to an acute heat shock at 42 or $45^{\circ} \mathrm{C}$ followed by $16 \mathrm{~h}$ recovery at $37^{\circ} \mathrm{C}$. Whereas none of the tested HDAC inhibitors had an effect on cell viability under control conditions (Fig. 4b, c), they markedly improved cell survival upon exposure to 120 min of heat shock at $42^{\circ} \mathrm{C}$ (Fig. 4b) or 30 min of heat shock at $45{ }^{\circ} \mathrm{C}$ (Fig. 4c). Cells treated with HDAC inhibitors and exposed to heat shock at $42{ }^{\circ} \mathrm{C}$ were more resistant to stress than the non-treated cells both immediately after heat shock (decrease in cell death by $29 \%$ for BGP-15, $52 \%$ for TSA, and 33\% for VPA) and during the recovery (decrease in cell death by $29 \%$ for BGP-15, 41\% for TSA, and 25\% for VPA). Furthermore, HDAC inhibition offered cytoprotection upon exposure to a

HDAC inhibition protects cells against proteotoxic stress We hypothesized that increased chromatin accessibility by HDAC inhibition enhances the HSR. Thus, we analysed the 
A

HSPA1A/B

DNAJB1
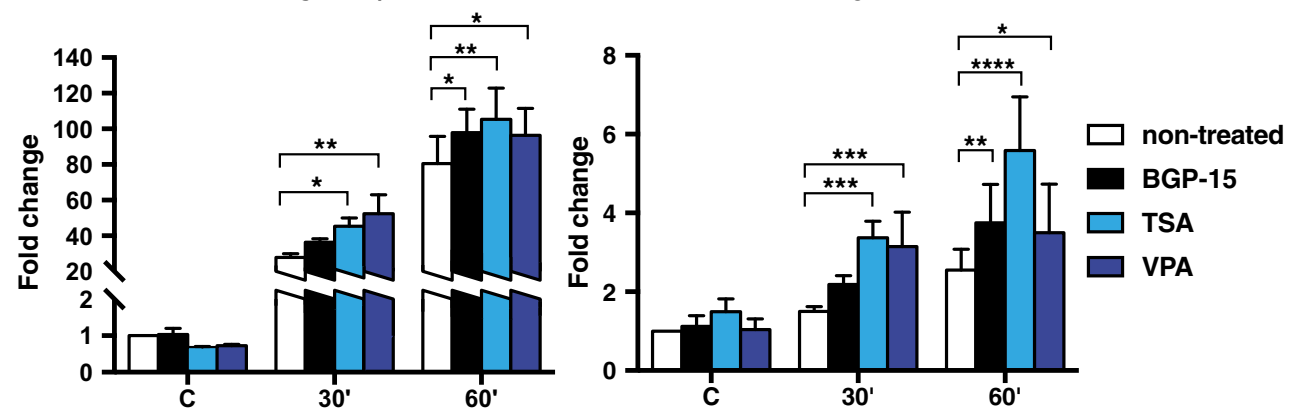

B

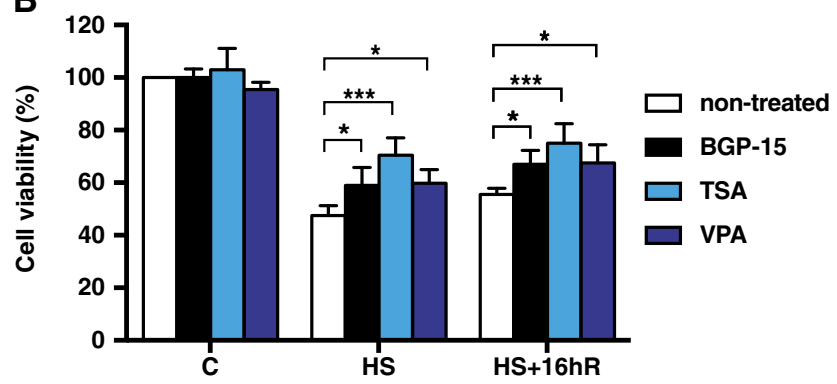

C

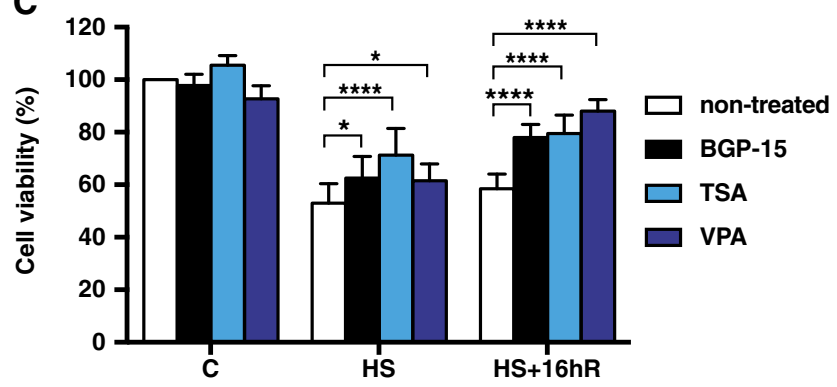

Fig. 4 HDAC inhibition protects cells against proteotoxic stress. a MEFs were pre-treated with $10 \mu \mathrm{M}$ BGP-15, $10 \mu \mathrm{M}$ TSA, $500 \mu \mathrm{M}$ VPA, or left untreated for $1 \mathrm{~h}$ and then exposed to heat shock at $42{ }^{\circ} \mathrm{C}$ for indicated times, or left at $37^{\circ} \mathrm{C}(\mathrm{C})$. The mRNA levels of HSPA1A/B $(H s p 70)$ and $D N A J B 1$ (Hsp40) were quantified using qRT-PCR and normalized against RNA18S5. The values are shown relative to the respective mRNA levels in the non-treated cells under control conditions $(C)$, which was arbitrarily set to value 1 . The data are presented as mean values from four independent experiments plus the SEM. b, c MEFs were pre-treated

severe 30-min heat shock at $45{ }^{\circ} \mathrm{C}$, both immediately after heat shock (decrease in cell death by $29 \%$ for BGP-15, $53 \%$ for TSA, and $19 \%$ for VPA) and during the recovery (decrease in cell death by $37 \%$ for BGP-15, $41 \%$ for TSA, and $50 \%$ for VPA) (Fig. 4c). Taken together, our results revealed that HDAC inhibitors enhance the HSR and improve cell survival upon proteotoxic stress.

\section{Discussion}

Despite numerous attempts to develop strategies for chemical activation of the HSF1 and HSF1-mediated heat shock response (HSR), no effective pharmacological interventions are currently in clinical use. Previous chemical screens with $10 \mu \mathrm{M}$ BGP-15, $10 \mu \mathrm{M}$ TSA, $500 \mu \mathrm{M}$ VPA, or left untreated for $1 \mathrm{~h}$ and then exposed to heat shock $(H S)$ for $120 \mathrm{~min}$ at $42^{\circ} \mathrm{C}(\mathbf{b})$ or $30 \mathrm{~min}$ at $45^{\circ} \mathrm{C}(\mathbf{c})$ and left to recover for $16 \mathrm{~h}$ at $37^{\circ} \mathrm{C}(H S+16 h R)$. The amount of cell death was measured using Calcein AM dye, and the signal of nontreated, non-heat-shocked cells were set to value 0 . The data are presented as mean values from four independent experiments plus the SEM. a-c Statistical analyses were performed using repeated measures two-way ANOVA with Holm-Sidak post hoc test. $* P \leq 0.05 ; * * P \leq 0.01$; $* * * P \leq 0.001 ; * * * * P<0.0001$

focused on the activation of HSF1 in the absence of stress, which in most cases resulted in cytotoxicity. Here, by using BGP-15, we present for the first time the model of enhanced HSR through HDAC inhibition. Treatment with BGP-15 alone inhibits the activity of HDACs, which increases chromatin accessibility for components of the transcription machinery at multiple genomic loci including HSPA1A. We demonstrate that BGP-15 accelerates both the activation and attenuation of the HSF1 cycle upon stress. Moreover, BGP-15 sensitizes HSF1 by lowering its activation threshold, thereby enhancing expression of Hsps already at a febrile range of temperatures. We found that similarly to BGP-15, the wellknown potent HDAC inhibitors TSA and VPA enhance expression of Hsps, which improves cell survival upon exposure to proteotoxic stress. 
Fig. 5 A model for chaperone co-inducing effect of HDAC inhibitors on the heat shock response (HSR). Certain pathological conditions are associated with accumulation of misfolded proteins in cells without inducible expression of Hsps. Inhibition of HDACs increases chromatin accessibility and lowers the threshold for HSF1 activation, thereby enabling and enhancing the HSR. HATs histone acetyltransferases, HDACs histone deacetylases, HDAC $i$ HDAC inhibitors, HSPS heat shock proteins

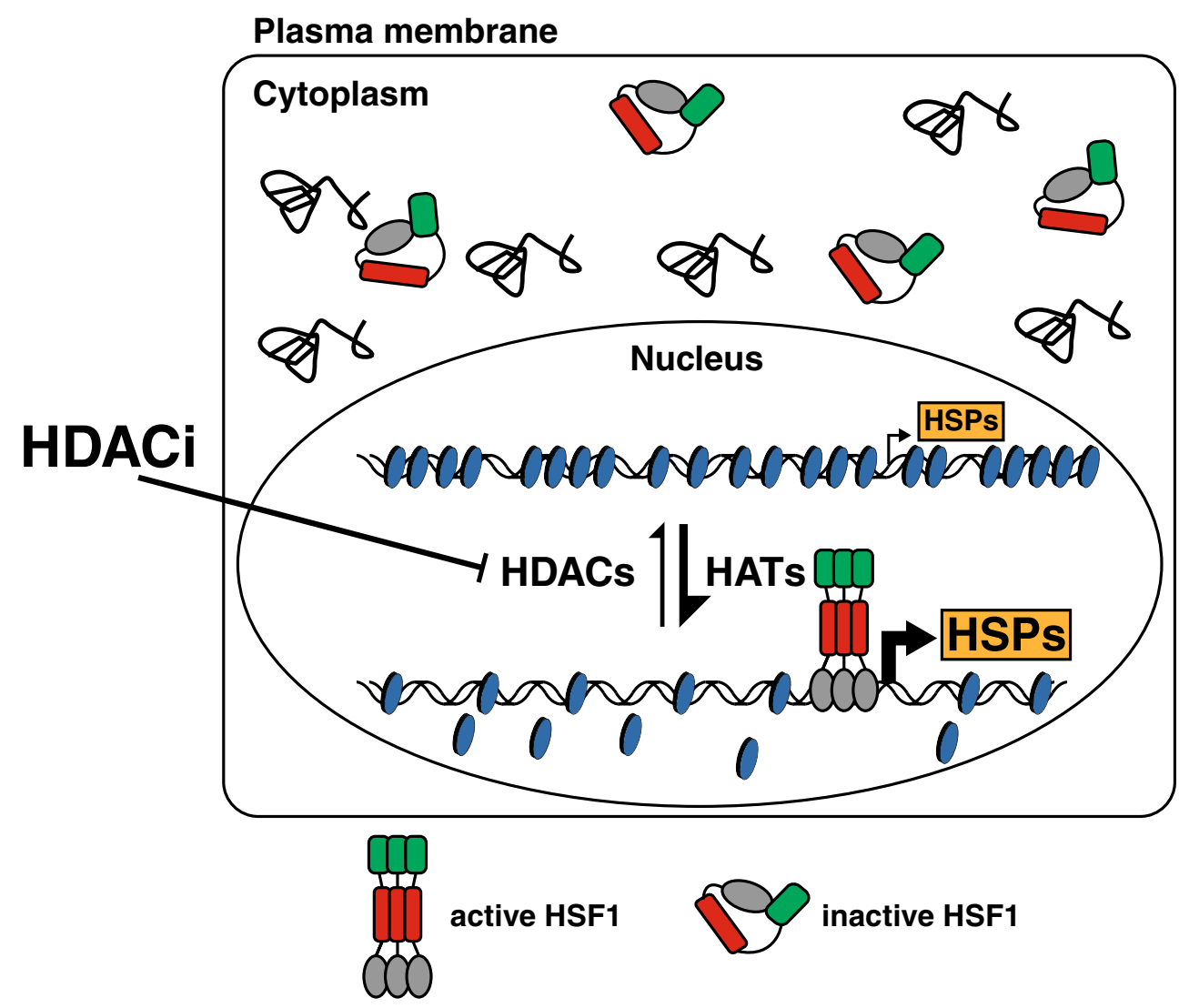

8 misfolded protein

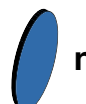

nucleosome
BGP-15 is a multi-target drug, with advantageous effects in a variety of proteinopathic disease models (Zhang et al. 2011; Gehrig et al. 2012; Eroglu et al. 2014; Henstridge et al. 2014; Salah et al. 2016). Human clinical trials in healthy individuals (Literáti-Nagy et al. 2009, 2012) and insulin-resistant nondiabetic patients (Literáti-Nagy et al. 2009) demonstrated an excellent safety profile indicating therapeutic potential presumably through Hsp induction. However, BGP-15 not only increases chaperone expression, but it also restores mitochondrial function and inhibits PARP-1 and TNF- $\alpha$-induced signalling pathways (Chung et al. 2008; Gombos et al. 2011; Henstridge et al. 2014; Gungor et al. 2014; Sumegi et al. 2017). Previously, these effects of BGP-15 were linked to BGP-15-induced remodelling of lipid rafts and fluidization of the membranes (Gombos et al. 2011). In this study, we expand the knowledge of the BGP-15 mode of action by showing that BGP-15 can also interfere with intracellular proteins, such as HDACs, which results in chromatin rearrangement. This novel mode of action of BGP-15 provides an additional explanation for its chaperone co-inducing activity that has been reported in a variety of disease models (Zhang et al. 2011; Gehrig et al. 2012; Eroglu et al. 2014; Henstridge et al. 2014; Salah et al. 2016). Compared with TSA, BGP-15 is less efficient in inhibiting the activity of HDACs in the cellular context, as well as BGP-15 is unable to inhibit the activity of purified HDACs (Fig. 2). The precise mechanism how BGP-15 inhibits HDAC activity is currently unknown. It has been shown that HDACs function as the catalytic core of multi-protein complexes, such as CoREST or the nuclear receptor co-repressor (NCoR) complex (Millard et al. 2017). Within these multi-protein complexes, the HDAC activity is often regulated by the presence of additional HDACs. For example, HDAC4 is essential for the deacetylation activity and transcriptional repression of the NCoR-SMRT-HDAC3 complex (Fischle et al. 2002). As multiple non-HDAC targets have been described for hydroxamate inhibitors (Bantscheff et al. 2011), we speculate that BGP-15 might target nonHDAC subunits and as such could interfere with the formation and activity of the multi-protein HDAC complexes (Millard et al. 2017). Currently, capture compound experiments (Köster et al. 2007), consisting of a biotin-labelled BGP-15, that allow immunoprecipitation of BGP-15 and identification of its interaction partners via mass spectrometry are being pursued in our laboratory.

Being a multi-target drug, it is plausible that the chaperone co-inducing property of BGP-15 consists of combinatorial 
effects occurring in the plasma membrane and intracellular compartments. Several diseases are described as a network phenomenon in which the partial inhibition of a small number of targets can be more efficient than the complete inhibition of a single target (Csermely et al. 2005; Csermely et al. 2013). Future studies should address the mechanisms by which BGP15 intervenes with these processes.

Under normal conditions, the expression of Hsps is regulated in an HSF1-independent manner (McMillan et al. 1998; Mahat et al. 2016). Upon proteotoxic stress, HSF1 is activated to bind to its target promoters, which initiates a cascade of events, leading to a release of the paused RNA polymerase II and enabling inducible gene expression (Lis and Wu 1993). Chromatin accessibility at the target promoters of HSF1 influences its ability to recognize and bind DNA (Labbadia and Morimoto 2015; Leach et al. 2016). In all eukaryotes, chromatin accessibility depends on nucleosome occupancy and positioning, controlled by a myriad of histone tail modifications, such as acetylation and methylation (Tessarz and Kouzarides 2014). Acetylation of histones is dynamically regulated by histone acetyltransferases (HATs), enhancing recruitment of the chromatin remodelling complexes, e.g. SWI/SNF, which facilitates the removal of nucleosomes, thereby increasing chromatin accessibility for transcription factors (Tessarz and Kouzarides 2014). Since HDACs counteract acetylation of histones, they maintain compact chromatin conformation and transcriptional silencing. Inhibition of HDACs leads to hyperacetylation of histones followed by their removal from chromatin. Based on our findings that BGP-15 acts as an HDAC inhibitor and increases chromatin accessibility, we propose a model where HDAC inhibition results in enhanced HSR in the event of proteotoxic insults (Fig. 5). Importantly, increased chromatin accessibility lowers the threshold for HSF1 activation and accelerates HSF1 binding to its target promoters. This capacity of lowering the threshold can be used to activate the HSR under those conditions when it is not normally activated (Fig. 1e). Interestingly, the Hsp co-inducing effect of BGP-15 was detected already at $15 \mathrm{~min}$ of heat stress for $D N A J B 1$ mRNA (Fig. 1d), whereas for HSPA1A/B mRNA at $30 \mathrm{~min}$ of stress. This observation raises the question how these genes respond differently to BGP-15 treatment. A recent study in MEFs showed that the $D N A J B 1$ promoter is already occupied by HSF1 under control conditions whereas the HSPA1A and HSPA1B promoters are not (Mahat et al. 2016). Thus, as the DNAJB1 promoter is occupied by HSF1 before stress, treatment with BGP-15 could increase the pre-existence binding of HSF1 in that specific locus, which would not be the case for HSPA1A and HSPA1B promoters. Alternatively, the difference in the kinetics of inducible expression of HSPA1A/B and DNAJB1 could provide an explanation for observed phenomena. $H S P A 1 A / B$ are unique genes with rapid activation kinetics (Zobeck et al. 2010), which result in a much higher increase of mRNA upon stress when compared with $D N A J B 1$ (20-fold vs. 0.5 -fold) as shown by our results. Assuming that the transcription of $H S P A 1 A / B$ is already functioning close to the maximal rate during the initial phase of HSR, the beneficial effect of BGP-15 would take a longer time to occur.

It is also worth noticing that enhanced expression of Hsps due to combined heat stress and HDAC inhibition was already shown a decade ago in model organisms Xenopus (Ovakim and Heikkila 2003) and Drosophila (Zhao et al. 2006). Moreover, increased Hsp levels were found in rodents treated with HDAC inhibitors and exposed to various neurological stresses, such as ischaemia (Faraco et al. 2006; Xuan et al. 2012), haemorrhage (Sinn et al. 2007), spinal cord injury (Lv et al. 2011), and middle cerebral artery occlusion (Sinn et al. 2007). However, the mechanism underlying the enhanced HSR by HDAC inhibition has not previously been reported.

HDAC inhibitors belong to a large and diverse family of compounds with beneficial effects in a wide range of animal disease models, including glutamate excitotoxicity (Marinova et al. 2009), chronic pain (Wang et al. 2016), and atrial fibrillation (Lkhagva et al. 2016). Furthermore, chromatin modifiers and especially HDAC inhibitors are currently used as drugs for human diseases, such as cancer (Roche and Bertrand 2016), diabetes (Arguelles et al. 2016), and neurological disorders (Falkenberg and Johnstone 2014). Our finding that BGP-15 acts as an HDAC inhibitor and that HDAC inhibition, in general, enhances the HSR presents a new approach to restore protein homeostasis in protein folding diseases.

Acknowledgements We thank Jenny Joutsen for constructive discussions and critical review of the manuscript and Malin Blom for expert help with the ChIP assay. This study was financially supported by the Academy of Finland, the Sigrid Jusélius Foundation, the Magnus Ehrnrooth Foundation, the Finnish Cancer Organisations, and the Åbo Akademi University Foundation (LS), Hungarian Scientific Research Fund grant OTKA NN 111006 (LV), the Turku Doctoral Program of Biomedical Sciences, the Magnus Ehrnrooth Foundation, and Instrumentarium Science Foundation (MAB), EMBO short-term fellowship and Hungarian Basic Research Fund grant MB08-B (TC), K. Albin Johanssons Foundation (SVH), and FEBS short-term fellowship (NT).

\section{Compliance with ethical standards}

Conflict of interests The authors declare that they have no competing interests.

Open Access This article is distributed under the terms of the Creative Commons Attribution 4.0 International License (http:// creativecommons.org/licenses/by/4.0/), which permits unrestricted use, distribution, and reproduction in any medium, provided you give appropriate credit to the original author(s) and the source, provide a link to the Creative Commons license, and indicate if changes were made.

\section{References}

Arguelles AO, Meruvu S, Bowman JD, Choudhury M (2016) Are epigenetic drugs for diabetes and obesity at our door step? Drug Discov Today 21:499-509. doi:10.1016/j.drudis.2015.12.001 
Bantscheff M, Hopf C, Savitski MM et al (2011) Chemoproteomics profiling of HDAC inhibitors reveals selective targeting of HDAC complexes. Nat Biotechnol 29:255-265. doi:10.1038/nbt.1759

Bitterman KJ, Anderson RM, Cohen HY et al (2002) Inhibition of silencing and accelerated aging by nicotinamide, a putative negative regulator of yeast Sir2 and human SIRT1. J Biol Chem 277:4509945107. doi:10.1074/jbc.M205670200

Budzyński M, Sistonen L (2017) Versatile functions of heat shock factors: It is not all about stress. Curr Immunol Rev. doi:10.2174/ 1573395513666170316110147

Budzyński MA, Puustinen MC, Joutsen J, Sistonen L (2015) Uncoupling stress-inducible phosphorylation of heat shock factor 1 from its activation. Mol Cell Biol 35:2530-2540. doi:10.1128/MCB.00816-14

Calamini B, Silva MC, Madoux F et al (2011) Small-molecule proteostasis regulators for protein conformational diseases. Nat Chem Biol 8:185-196. doi:10.1038/nchembio.763

Chen Y, Wang B, Liu D et al (2014) Hsp90 chaperone inhibitor 17-AAG attenuates $\mathrm{A} \beta$-induced synaptic toxicity and memory impairment. $\mathrm{J}$ Neurosci 34:2464-2470. doi:10.1523/JNEUROSCI.0151-13.2014

Chitnis A, Balle-Cuif L (2016) The Notch meeting: an odyssey from structure to function. Development 143:547-553. doi:10.1242/dev.131086

Chung J, Nguyen A-K, Henstridge DC et al (2008) HSP72 protects against obesity-induced insulin resistance. Proc Natl Acad Sci U S A 105:1739-1744. doi:10.1073/pnas.0705799105

Crul T, Toth N, Piotto S, Literati-Nagy P, Tory K, Haldimann P et al. (2013) Hydroximic acid derivatives: pleiotropic HSP co-inducers restoring homeostasis and robustness. Curr Pharm Des 19(3):309346.

Csermely PP, Ágoston V, Pongor S (2005) The efficiency of multi-target drugs: the network approach might help drug design. Trends Pharmacol Sci 26:178-182. doi:10.1016/j.tips.2005.02.007

Csermely P, Korcsmáros T, Kiss HJM et al (2013) Structure and dynamics of molecular networks: a novel paradigm of drug discovery: a comprehensive review. Pharmacol Ther 138:333-408

Cuatrecasas P, Fuchs S, Anfinsen CB (1967) Catalytic properties and specificity of the extracellular nuclease of Staphylococcus aureus. J Biol Chem 242:1541-1547. doi:10.1038/nrd2906

Delcuve GP, Khan DH, Davie JR (2012) Roles of histone deacetylases in epigenetic regulation: emerging paradigms from studies with inhibitors. Clin Epigenetics 4:5. doi:10.1186/1868-7083-4-5

Elsing AN, Aspelin C, Björk JK et al (2014) Expression of HSF2 decreases in mitosis to enable stress-inducible transcription and cell survival. J Cell Biol 206:735-749. doi:10.1083/jcb.201402002

Eroglu B, Kimbler DE, Pang J et al (2014) Therapeutic inducers of the HSP70/HSP110 protect mice against traumatic brain injury. J Neurochem 130:626-641. doi:10.1111/jnc.12781

Escribá PV, Busquets X, Inokuchi J et al (2015) Membrane lipid therapy: modulation of the cell membrane composition and structure as a molecular base for drug discovery and new disease treatment. Prog Lipid Res 59:38-53. doi:10.1016/j.plipres.2015.04.003

Falkenberg KJ, Johnstone RW (2014) Histone deacetylases and their inhibitors in cancer, neurological diseases and immune disorders. Nat Rev Drug Discov 13:673-691. doi:10.1038/nrd4360

Faraco G, Pancani T, Formentini L et al (2006) Pharmacological inhibition of histone deacetylases by suberoylanilide hydroxamic acid specifically alters gene expression and reduces ischemic injury in the mouse brain. Mol Pharmacol 70:1876-1884. doi:10.1124/mol.106.027912

Fischle W, Dequiedt F, Hendzel MJ et al (2002) Enzymatic activity associated with class II HDACs is dependent on a multiprotein complex containing HDAC3 and SMRT/N-CoR. Mol Cell 9:45-57

Gao L, Cueto MA, Asselbergs F, Atadja P (2002) Cloning and functional characterization of HDAC11, a novel member of the human histone deacetylase family. J Biol Chem 277:25748-25755. doi:10.1074/ jbc.M111871200
Gehrig SM, van der Poel C, Sayer TA et al (2012) Hsp72 preserves muscle function and slows progression of severe muscular dystrophy. Nature 484:394-398. doi:10.1038/nature10980

Gombos I, Crul T, Piotto S et al (2011) Membrane-lipid therapy in operation: the HSP co-inducer BGP-15 activates stress signal transduction pathways by remodeling plasma membrane rafts. PLoS One 6: e28818. doi:10.1371/journal.pone.0028818

Göttlicher M, Minucci S, Zhu P et al (2002) Valproic acid defines a novel class of HDAC inhibitors inducing differentiation of transformed cells. EMBO J 20:6969-6978. doi:10.1093/emboj/20.24.6969

Gungor B, Gombos I, Crul T et al (2014) Rac1 participates in thermally induced alterations of the cytoskeleton, cell morphology and lipid rafts, and regulates the expression of heat shock proteins in B16F10 melanoma cells. PLoS One 9:e89136. doi:10.1371/journal.pone.0089136

Hargitai J, Lewis H, Boros I et al (2003) Bimoclomol, a heat shock protein co-inducer, acts by the prolonged activation of heat shock factor-1. Biochem Biophys Res Commun 307:689-695. doi:10. 1016/S0006-291X(03)01254-3

Hartl FU, Bracher A, Hayer-Hartl M (2011) Molecular chaperones in protein folding and proteostasis. Nature 475:324-332. doi:10. 1038/nature 10317

Henikoff S, Shilatifard A (2011) Histone modification: cause or cog? Trends Genet 27:389-396. doi:10.1016/j.tig.2011.06.006

Henstridge DC, Bruce CR, Drew BG et al (2014) Activating HSP72 in rodent skeletal muscle increases mitochondrial number and oxidative capacity and decreases insulin resistance. Diabetes 63:18811894. doi: $10.2337 / \mathrm{db} 13-0967$

Hietakangas V, Ahlskog JK, Jakobsson AM et al (2003) Phosphorylation of serine 303 is a prerequisite for the stress-inducible SUMO modification of heat shock factor 1. Mol Cell Biol 23:2953-2968. doi: 10.1128/MCB.23.8.2953-2968.2003

Kennedy TL, Swiderski K, Murphy KT et al (2016) BGP-15 improves aspects of the dystrophic pathology in mdx and dko mice with differing efficacies in heart and skeletal muscle. Am J Pathol 186: 3246-3260. doi:10.1016/j.ajpath.2016.08.008

Köster H, Little DP, Luan P et al (2007) Capture compound mass spectrometry: a technology for the investigation of small molecule protein interactions. Assay Drug Dev Technol 5:381-390. doi:10.1089/ adt.2006.039

Labbadia J, Morimoto RII (2015) Repression of the heat shock response is a programmed event at the onset of reproduction. Mol Cell 59: 639-650. doi:10.1016/j.molcel.2015.06.027

Leach MD, Farrer RA, Tan K et al (2016) Hsf1 and Hsp90 orchestrate temperature-dependent global transcriptional remodelling and chromatin architecture in Candida albicans. Nat Commun 7:11704. doi: 10.1038/ncomms 11704

Lindsay CR, Morozov VM, Ishov AM (2008) PML NBs (ND10) and Daxx: from nuclear structure to protein function. Front Biosci 13: $7132-7142$

Lis J, Wu C (1993) Protein traffic on the heat shock promoter: parking, stalling, and trucking along. Cell 74:1-4

Literáti-Nagy B, Kulcsár E, Literáti-Nagy Z et al (2009) Improvement of insulin sensitivity by a novel drug, BGP-15, in insulin-resistant patients: a proof of concept randomized double-blind clinical trial. Horm Metab Res 41:374-380. doi:10.1055/s-0028-1128142

Literáti-Nagy Z, Tory K, Literáti-Nagy B et al (2012) The HSP coinducer BGP-15 can prevent the metabolic side effects of the atypical antipsychotics. Cell Stress Chaperones 17:517-521. doi:10. 1007/s12192-012-0327-5

Literáti-Nagy B, Tory K, Peitl B et al (2014) Improvement of insulin sensitivity by a novel drug candidate, BGP-15, in different animal studies. Metab Syndr Relat Disord 12:125-131. doi:10.1089/met. 2013.0098

Lkhagva B, Kao Y-H, Chen Y-C et al (2016) Targeting histone deacetylases: a novel therapeutic strategy for atrial fibrillation. Eur J Pharmacol 781:250-257. doi:10.1016/j.ejphar.2016.04.034 
Lv L, Sun Y, Han X et al (2011) Valproic acid improves outcome after rodent spinal cord injury: potential roles of histone deacetylase inhibition. Brain Res 1396:60-68. doi:10.1016/j.brainres.2011.03.040

Mahat DB, Salamanca HH, Duarte FM et al (2016) Mammalian heat shock response and mechanisms underlying its genome-wide transcriptional regulation. Mol Cell 62:63-78. doi:10.1016/j.molcel. 2016.02.025

Manova V, Singh SK, Iliakis G (2012) Processing of DNA double strand breaks by alternative non-homologous end-joining in hyperacetylated chromatin. Genome Integr 3:4. doi:10.1186/2041-9414-3-4

Marinova Z, Ren M, Wendland JR et al (2009) Valproic acid induces functional heat-shock protein 70 via Class I histone deacetylase inhibition in cortical neurons: a potential role of $\mathrm{Sp} 1$ acetylation. J Neurochem 111:976-987. doi:10.1111/j.1471-4159.2009.06385.x

McMillan DR, Xiao X, Shao L et al (1998) Targeted disruption of heat shock transcription factor 1 abolishes thermotolerance and protection against heat-inducible apoptosis. J Biol Chem 273:7523-7528. doi:10.1074/jbc.273.13.7523

Millard CJ, Watson PJ, Fairall L, Schwabe JWR (2017) Targeting class I histone deacetylases in a "complex" environment. Trends Pharmacol Sci. doi:10.1016/j.tips.2016.12.006

Neef DW, Turski ML, Thiele DJ (2010) Modulation of heat shock transcription factor 1 as a therapeutic target for small molecule intervention in neurodegenerative disease. PLoS Biol 8:e1000291. doi:10. 1371/journal.pbio.1000291

Ovakim DH, Heikkila JJ (2003) Effect of histone deacetylase inhibitors on heat shock protein gene expression during Xenopus development. Genesis 36:88-96. doi:10.1002/gene.10202

Perry MD, Aujame L, Shtang S, Moran LA (1994) Structure and expression of an inducible HSP70-encoding gene from Mus musculus. Gene 146:273-278. doi:10.1016/0378-1119(94)90305-0

Raychaudhuri S, Loew C, Körner R et al (2014) Interplay of acetyltransferase EP300 and the proteasome system in regulating heat shock transcription factor 1. Cell 156:975-985. doi:10.1016/j.cell.2014. 01.055

Roche J, Bertrand P (2016) Inside HDACs with more selective HDAC inhibitors. Eur J Med Chem 121:451-483. doi:10.1016/j.ejmech. 2016.05.047

Salah H, Li M, Cacciani N, et al (2016) The chaperone co-inducer BGP15 alleviates ventilation-induced diaphragm dysfunction. Sci Transl Med 8:350ra103. doi:10.1126/scitranslmed.aaf7099

Sengupta N, Seto E (2004) Regulation of histone deacetylase activities. J Cell Biochem 93:57-67. doi:10.1002/jcb.20179

Sharma S, Mishra R, Walker BL et al (2014) Celastrol, an oral heat shock activator, ameliorates multiple animal disease models of cell death. Cell Stress Chaperones 20:185-201. doi:10.1007/s12192-0140536-1

Simons K, Toomre D (2000) Lipid rafts and signal transduction. Nat Rev Mol Cell Biol 1:31-39. doi:10.1038/35036052
Sinn D-I, Kim S-J, Chu K et al (2007) Valproic acid-mediated neuroprotection in intracerebral hemorrhage via histone deacetylase inhibition and transcriptional activation. Neurobiol Dis 26:464-472. doi: 10.1016/j.nbd.2007.02.006

Su K-H, Dai C (2016) Metabolic control of the proteotoxic stress response: implications in diabetes mellitus and neurodegenerative disorders. Cell Mol Life Sci 73:4231-4248. doi:10.1007/s00018-0162291-1

Sumegi K, Fekete K, Antus C et al (2017) BGP-15 protects against oxidative stress- or lipopolysaccharide-induced mitochondrial destabilization and reduces mitochondrial production of reactive oxygen species. PLoS One 12:e0169372. doi:10.1371/journal.pone. 0169372

Tessarz P, Kouzarides T (2014) Histone core modifications regulating nucleosome structure and dynamics. Nat Rev Mol Cell Biol 15: 703-708. doi:10.1038/nrm3890

Vígh L, Literáti PN, Horváth I et al (1997) Bimoclomol: a nontoxic, hydroxylamine derivative with stress protein-inducing activity and cytoprotective effects. Nat Med 3:1150-1154. doi:10.1038/ nm1097-1150

Wang W, Cui S, Lu R, Zhang H (2016) Is there any therapeutic value for the use of histone deacetylase inhibitors for chronic pain? Brain Res Bull 125:44-52. doi:10.1016/j.brainresbull.2016.04.010

West JD, Wang Y, Morano KA (2012) Small molecule activators of the heat shock response: chemical properties, molecular targets, and therapeutic promise. Chem Res Toxicol 25:2036-2053. doi:10. $1021 /$ tx300264x

Westerheide SD, Anckar J, Stevens SM et al (2009) Stress-inducible regulation of heat shock factor 1 by the deacetylase SIRT1. Science 323:1063-1066. doi:10.1126/science.1165946

Xuan A, Long D, Li J et al (2012) Neuroprotective effects of valproic acid following transient global ischemia in rats. Life Sci 90:463-468. doi:10.1016/j.1fs.2012.01.001

Yoshidas M, Yoshida M, Kijima M et al (1990) Potent and specific inhibition of mammalian histone deacetylase both in vivo and in vitro by trichostatin A. J Biol Chem 265:17174-17179

Zelin E, Freeman BC (2015) Lysine deacetylases regulate the heat shock response including the age-associated impairment of HSF1. J Mol Biol 427:1644-1654. doi:10.1016/j.jmb.2015.02.010

Zhang D, Ke L, Mackovicova K et al (2011) Effects of different small HSPB members on contractile dysfunction and structural changes in a Drosophila melanogaster model for atrial fibrillation. J Mol Cell Cardiol 51:381-389. doi:10.1016/j.yjmec.2011.06.008

Zhao YM, Chen X, Sun H et al (2006) Effects of histone deacetylase inhibitors on transcriptional regulation of the hsp70 gene in Drosophila. Cell Res 16:566-576. doi:10.1038/sj.cr.7310074

Zobeck KL, Buckley MS, Zipfel WR, Lis JT (2010) Recruitment timing and dynamics of transcription factors at the Hsp70 loci in living cells. Mol Cell 40:965-975. doi:10.1016/j.molcel.2010.11.022 